\title{
Modeling PN Modulation In-Car Laser Radar for Range and Velocity Measurement
}

\author{
Shiwei $\mathrm{Li}^{1,}$ a , Xiangcheng Chen ${ }^{2, b^{*}}$ and Xuesong Mao ${ }^{3, \mathrm{c}}$ \\ ${ }^{1}$ School of Aeronautics'Northwestern Polytechnical University, Xi'an, China \\ ${ }^{2}$ HeFei University of Technology, Hefei, China \\ ${ }^{3}$ Wuhan University of Science and Technology, Wuhan, China \\ alisw204@163.com, ${ }^{\mathrm{b}}$ corresponding author :chenxgcg@ustc.edu, ${ }^{\mathrm{c}}$ xsmao@wust.edu.cn
}

Keywords: Laser Radar; PN modulation; FFT;. correlation function.

\begin{abstract}
A novel algorithm for range and velocity measurement in Pulse Doppler Laser Radar was proposed in this paper. We used pseudo-random code to modulate the $1.55 \mu \mathrm{m}$ continuous laser in the laser Radar transmitter firstly, and then different detection method was utilized for sensing target range and target velocity. While our algorithm employed direction detection method to obtain the target range, coherence detection method was used to get the target speed simultaneously in the Laser Radar receiver. Compared to previous method, our method modulate $\mathrm{CW}$ laser with pseudonoise (PN) code directly. Target range is obtained by calculating the cross correlation function between the PN Code and the echo signal of direct detection, and FFT is used on the echo signal of coherence detection directly to calculate the Doppler frequency. The maximum measurable speed can be increased to $240 \mathrm{~km} / \mathrm{h}$ by this way. Moreover, the length of the transmitted signal is several microseconds, which make the possibility of realizing a high scanning speed in automotive applications.
\end{abstract}

\section{Introduction}

LASER radar is a valuable tool in many applications such as civilian and military applications[1, 2], due to the ability of three dimensional imaging of intensity, velocity, range. While commercial in-car laser radars provide better spatial resolution than millimeter-wave radar due to high directivity of the laser beam, they have the shortcomings of poor performance to approximate the target speed by range differentiation, which has the problem of excessive time consumption and the introduction of large errors. To improve the target speed detection accuracy, a better method is to measure the Doppler frequency of the incoming signal[3,4].

For in-car laser radar, an importance factor is real-time measurement, and current commercial high-scan-speed in-car laser radar can make measurements at more than 20000 times per second. Using the traditional frequency modulation continuous-wave (FMCW) method, NASA provided a laser radar system to aid space exploration, which measures target range and velocity with high precision $(1 \mathrm{~cm}$ and $1 \mathrm{~cm} / \mathrm{s}$, respectively). Unfortunately, the period of the transmission signal used exceeds $6 \mathrm{~ms}$, which cannot satisfy the real-time requirement[3]. Another factor for in-car laser radar is the cost. Recently, Gao\&[5] proposed a new FMCW approach utilizing the measurement of complex-optical-field for range and velocity measurement with high accuracy $(3.2 \mathrm{~cm}$ and $0.5 \mathrm{~m} / \mathrm{s})$. However, the cost of electro-optic I/Q modulator used in this method is very expensive and the calculating quantity is so large that it's difficult for this method to be applied in in-car laser radar.

Pulsed Doppler radar[6, 7]may be a good choice to gain the velocity and range simultaneously, where optical pulses are intensity modulated at a constant frequency before launched out through a telescope in the transmitter. When the target is moving, the reflected signal acquires a Doppler frequency shift. This reflected optical signal is detected by an optical receiver, and the frequency carried on the signal can be obtained through a Fourier process the target velocity can be determined. The velocity of the Doppler frequency shift can be either positive or negative depending on the 
direction of the velocity (moving toward or away from the observer). Nevertheless, dilemma has to be considered as the method used in pulsed Doppler radar, that is, a good choice of PRF (Pulse Repetition Frequency) to achieve a large unambiguous range will be a poor choice to achieve a large unambiguous velocity[5]. Thus, in traditional pulsed Doppler laser radar system, a tradeoff between the velocity resolution and ranges resolution has to be made.

Mao\& [3, 4] provided a new scheme to solve the dilemma existed in traditional pulsed Doppler laser radar, which modulated the transmitted laser using a composited series combining a PN code with periodic code by OR logic. Range measurement is obtained by multiplying the light speed by half the time difference between the transmitted and received signals. Received signal is buried in the thermal noise of the radar receiver and is invisible. Thus, a correlation function is used to locate the position of the received signal[8,9]. In order to calculate the speed information, this paper employing periodic code. While this method does have some advance in real-time performance, some shortcomings should also be taken into account. On the one hand, the $\varepsilon$-function like correlation characteristic of the PN code will be destroyed due to the periodic code, because the peak of the autocorrelation is broaden when periodic pulses are inserted into the PN code. On the other hand, the algorithm of Doppler frequency is to calculate the periodic pulsed echo signals, which decreases the maximum measure speed. Moreover, the Doppler spectrum energy contained in the PN code is discarded by this way.

The objective of this paper is to demonstrate our newly developed Doppler laser radar system that can simultaneously measure target range and speed with high precision. In this paper, first, we propose a new algorithm for modeling in-car laser radar and a new modulation scheme for the transmitted signal so that the proposed laser radar can simultaneously measure the target range and speed. The length of the signal assures the feasibility of scanning at high speed. Then, we describe a signal processing method for obtaining the target range and speed from the received signal. Compared with previous Doppler radar, our method can significantly increase the maximum measurable speed. Simulation is performed to validate the proposed method.

This paper is organized as follows: In Section II theoretical background about the range measurement and velocity measurement are described. Section III performs the simulation about the proposed method, and also discusses the results. A conclusion is drawn in Section IV, finally.

\section{Theoretical Background}

\section{A. PN Modulation for Range Measurement}

According to Laser Radar equation[10], the output signal can be expressed as

$$
P_{\text {ter }}-\frac{\tau_{0} \tau_{\alpha}^{2} D_{n}^{2} P_{t}(d A) P_{t}}{R^{2} \theta_{n}\left(\theta_{t} R\right)^{2}}
$$

Where $\tau_{0}$ denotes the LADAR receiver optics transmission, $\tau_{a n}$ is the atmospheric transmission, $D_{R}$ is the diameter of the aperture of the LADAR receiver optics, $\rho_{t}$ is the target surface reflectivity, $d A$ is the effective target surface area, $P_{t}$ is the transmitted laser power in watts(W), $R$ is the range between laser RADAR system and the target, $\theta_{R}$ is the target surface angular dispersion in steradians( $\left.\mathrm{sr}\right), \theta_{t}$ is the beam width of the LADAR transmitter in radians(rad).

From above equation, you can find that the relationship between the transmitted power and received power is $P_{\text {ses }} \propto P_{l}$. Let the modulation signal $x(t) \propto P_{\mathrm{s}}$, output signal $y(t) \propto F_{\text {det }}$, and then $y(\hat{v}) \propto x(\mathrm{t})$. Suppose that the amplified coefficient between $y(t)$ and $x(t)$ is G, and if the noise and time delay are also considered, $y(t)$ can be expressed in a form

$$
y(0)=z * x(t-R / c)+n(t)
$$

Here, $c$ denotes the light velocity.

For RM-CW Ladar, the input signal takes the form

$$
x(t)=F_{0} \alpha(t)
$$


Here, $P_{0}$ is the $\mathrm{CW}$ laser power, and $a(t)$ is usually generated by RM code. Then, $a(t)$ is expressed in a series form

$$
a(b)-\sum a_{[}, a(t-i \Delta t)
$$

Where the modulator code $a_{l}=1$ ar 0 , and

$$
a(t)= \begin{cases}11 & \left\{\leq t^{r} \propto \Delta t\right) \\ 0 & a \hbar h e r w i s e\end{cases}
$$

As another expression of $a_{i}$, denote $a_{i}^{r}$ with element 1 and $-1(i=0, \ldots, A-1)$ connected with $a_{i}$ by $\mathrm{t}$ he relation $a_{l}^{x}=2 * a_{l}-1$. Code $a_{l}^{r}$ is called as bipolar code. The cross-correlation of $a_{i}$ and $a_{l}^{r}$ is give

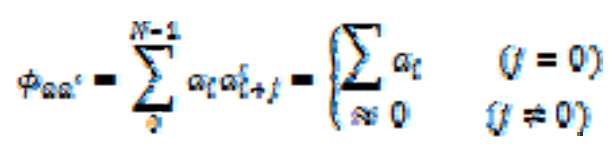

$n$ by

Now we expand input signal, the output signal, noise, in series form:

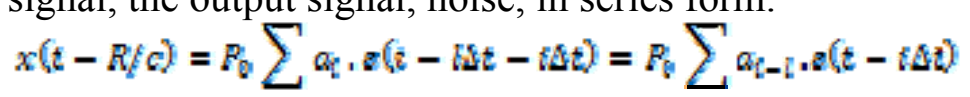

Here, $l=R z(\Delta t * c)$ denotes the echo delay.

$$
\begin{aligned}
& y(t)=\sum x \cdot a(t-i \Delta t) \\
& n(t)=\sum n_{t} \cdot r(t-i \Delta t)
\end{aligned}
$$

Then, Eq. (2) can be expressed as

$$
x=R_{0} G a_{l-l}+n_{l}
$$

The cross-correlation $S_{\mathrm{j}}$ of the signal $\gamma_{\mathrm{i}}$ with the modulation code $\alpha_{\mathrm{i}}^{r}$ as

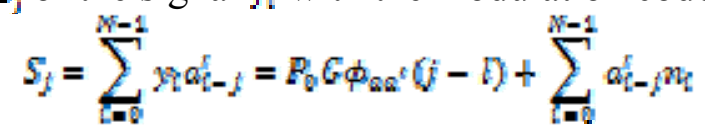

According to characteristics of $\phi_{a a^{\varepsilon}}$, the cross-correlation $s_{l}$ can be used to demodulation the range.

If the noise $n_{i}$ is independent of the modulation code $a_{i}$, the expectation value of $s_{i}$ is expressed according to Eq.(1) as

$$
E\left[S_{j}\right]=P_{0} G \phi_{\operatorname{ax}^{c}}(V-D)+\sum_{i=0}^{N-1} a_{l-j}^{r} \bar{n}
$$

Where $\bar{n}$ is ensemble average of $n_{i}$. The SNR equation is given in terms of the expectation value to the standard deviation of $s_{j}$.

$$
\operatorname{Far}\left[E_{j}\right] \approx \operatorname{var}\left(\sum_{=\mathbb{0}}^{N-1} \alpha_{[-j}^{r} m_{L}\right)-N * \operatorname{var}\left(m_{L}\right)
$$

\section{B. PN Modulation for Velocity Measurement}

For the CW laser radar, because of Doppler effect, echo signal frequency will be different to transmitted laser. Received light power at photosensitive surface of Photoelectric Detectors (PDs) can be expressed as:

$$
F^{\prime}=\left\{E_{1} \cos \left(2 \pi f t+\varphi_{1}\right)+E_{2} \cos \left(2 \pi f t+2 \pi f_{t} t+\varphi_{2}\right)\right\}^{2}
$$


Here, $E_{1}, E_{2}, f$ and $f_{D}$ represent the amplitude of the reference signal, the amplitude of the received signal, the optical frequency of the transmitting laser, and the Doppler frequency, respectively. $\sigma_{1}$ and $\varphi_{2}$ are the phase of the reference and received signals.

PDs have an upper limit cutoff frequency, which is rather low relative to the optical frequency; the output signal by the PDs can be expressed as:

$$
S_{i t}=2 E_{1} E_{2} \cos \left(2 \pi_{2 b} t+\varphi_{2}-\varphi_{1}\right)
$$

Here, subscript $b$ signifies that $s_{b}$ is a beat signal.

To obtain the Doppler frequency, Fourier analysis requires that the length of the beat signal cover at least one period of the sine wave expressed by (15). It's difficult for this method to obtain sufficient range information, thus, such a method cannot be adopted for in-car laser radar. Pulsed Doppler radar is a good choice to gain the velocity and range simultaneously. But the Doppler dilemma, which can also be expressed in a form $\nabla_{\max } R_{\max }=c \lambda / 8$, has to be considered when this method used in pulsed Doppler radar. Here, $\nabla_{\text {oxax }}$ denotes the maximum measurable Velocity, $R_{\text {vax }}$ is maximum unambiguous range, $c$ is light velocity, and $x$ is the laser wavelength. To break through the Doppler dilemma, one of the good choices is to modulate the amplitude of the transmitted signal by PN code [8]

$$
F^{\prime}=\left\{E_{1} \cos \left(2 \pi f t+\varphi_{1}\right)+c(t) E_{2} \cos \left(2 \pi f t+2 \pi f_{0} t+\varphi_{2}\right)\right\}^{2}
$$

Here, f̧z)is the continuous form of the modulation code and can be expressed by

$$
c(t)=\left\{\begin{array}{l}
0, \text { if } a\left(\left[\frac{\tau}{r_{\alpha}}\right]+1\right)=0 \\
1 . \text { if } c\left(\left[\frac{t}{r_{c}}\right]+1\right)=1
\end{array}\right.
$$

Where $\&(n)$ is the $n$th modulation code. Superscript $\sim$ signifies that the code sequence is an array, whose index is a nonnegative integer. [A] rounds the element $A$ to the nearest integer less than or equal to A. $\tau_{c}$ is the code width.

Similar to Eq(15), the output signal by the PDs for PN modulation Laser radar can be expressed as:

$$
S_{\bar{L} F}=2 c(t) E_{1} E_{2} \cos \left(2 \pi f t t+\varphi_{2}-\varphi_{1}\right)=S_{2}(t) * c(t)
$$

Eq.(15) tells us that, the output of the PN modulation laser radar is the output beat signal of CW Doppler laser radar multiply a continuous modulation code. In fact, $s_{b} F_{N}$ is not true in practice taking in considering of the optical wave propagation process in atmosphere. Thus, a more precise simulation model is a smooth curve, as is shown in Figure 1. Figure 1(a) shows original signal, and Figure 1(b) shows smoothing signal with a low pass-filter to simulate the wave propagation process in atmosphere.

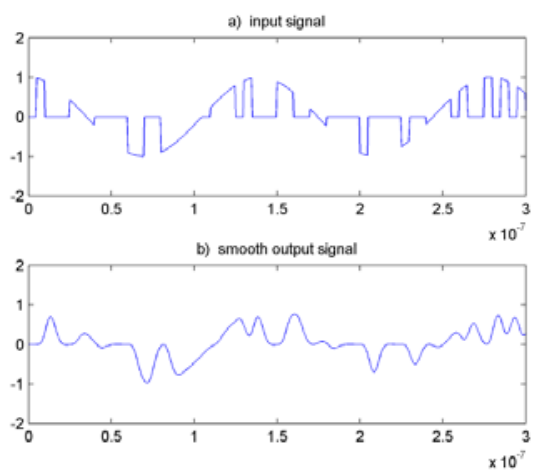

Fig 1 smoothing processing a)original input signal b)smoothing the input signal with low-pass filter 

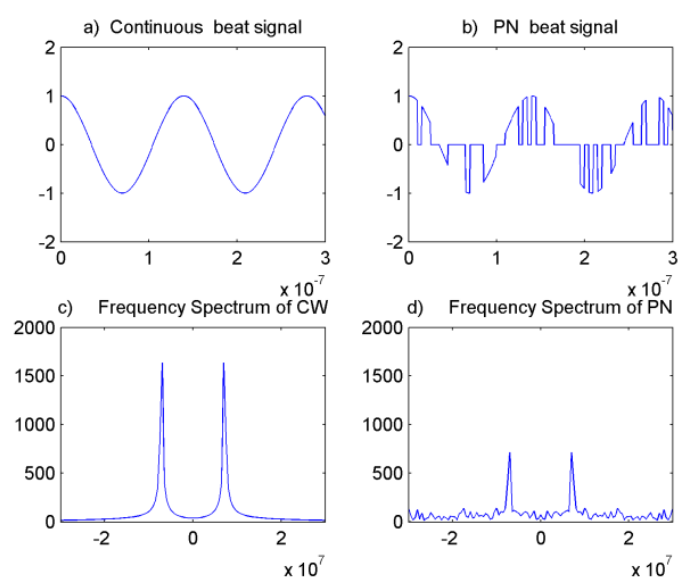

Fig 2 FFT for CW Doppler signal and PN modulation Doppler signal

Fourier analysis requires equidistant sampling as is told by mao\& [3, 4], but it does not mean the modulation code must contain period code. The Doppler frequency can be extracted directly using FFT on the coherent echo signal no matter whether the modulation code is periodic, and we demonstrate this as follow.

Consider a complex series $x(k)$ with $N$ samples of the form

Where $x$ is a complex number

$$
x_{\text {put }} x_{1, a+1} x_{k+\ldots, 2} x_{N-1}
$$$$
x_{\mathrm{l}}=x_{\mathrm{real}}+j x_{\text {mage }}
$$

Further, assume that that series outside the range $0, N-1$ is extended $N$-periodic, that is, $x_{k}=x_{k+\infty}$ for all time. The Fourier Transform of this series will be denoted $\mathcal{X ( k )}$, it will also have $N$ samples. The forward transform will be define as

$$
x(k)-\frac{1}{N} \sum_{0}^{W-1} x(k) e^{-\frac{\ln 2 \pi n}{N}}, n-0.1, \ldots n-1
$$

Now we set parts of the samples as 0 , the signal still contains the frequency we interest in. As is shown in Figure 2, While Figure 2(a) shows the CW Doppler echo beat signal and Figure 2(b) shows PN modulation echo beat signal, Figure 2(c) and Figure 2(d) show their frequency spectrum respectively. The Fourier Transform of the output beat signal of PN modulation Doppler echo signal has the same frequency but with different amplitude as the CW Doppler echo signal, due to the missing of the samples. This characteristic infers that we need not to combine a PN code sequence and a periodic pulse sequence by OR logic, as shown in Mao\& [3, 4], for the periodic pulse sequence will break the $\delta$-function like characteristics of cross-correlation between modulation code and echo signal of direct-detection, and it will also reduce the maximum measureable speed as shown in Mao\&[3, 4].

\section{The proposed PN Modulation Doppler Laser Radar}

The advantage of PN modulation for range measurement and velocity can fuse into one, so that the range and velocity can be obtained at the same time. The measurement schematic was shown in Figure 3. A polarized laser with a narrow linewidth is used as light source. The laser output is split into two branches by an optical splitter, of which one branch is used as reference signal, and the other is transmitted to an external modulator. The modulator modulates the amplitude of the laser using a modulation code sequence from a code generator and sends the modulated laser light to a collimator lens. The modulation part consists of the modulator and the code generator. The laser light sent to the collimator lens is passed through a $1 / 4 \lambda$ plate to transform linearly polarized light to circular polarized light, and then, is transmitted from the vehicle to gather range and speed information of the targets. The laser light reflected from the targets is passed through a $1 / 4 \lambda$ plate to transform circular polarized light to linearly polarized light firstly, and then, coupled to a fiber by a coupling lens. The received laser light is split into two parts, of which one is sent to a photodiode (PD) PD2 and the other 
to a coupler. The laser light sent to PD2 is converted into an electrical signal and is used to obtain the target range information. The laser sent to the coupler interferes with the reference signal and is sent to PD1. PD1 outputs the beat signal of the received and reference signals. Fourier analysis is used to obtain the Doppler frequency, and thus, the target speed information.

\section{Simulation and Discussion}

A PN modulation codes with 400 numbers was generated with a function named rand in MATLAB to generate uniformly distributed pseudorandom numbers between 0 and 1 firstly. After then, a threshold 0.5 was set to binarize the pseudorandom numbers. We use this code to modulate the CW laser, code width $\tau_{\varepsilon}=5 \mathrm{~ms}$, thus total modulation length equals $L=2 \mu \varepsilon, f_{\varepsilon}=100 \mathrm{~km} / \mathrm{h}$, the sampling rate $f=1 \mathrm{GHz}$.

Figure 4(a) shows the modulation function, and Figure 4(b) shows the simulation echo signal from PD1. The range is calculated by decoding the PD1 signal with bipolar code of modulation function. Figure 5 shows the cross-correlation function between PD1 echo signal and bipolar modulation codes. The correlation peak indicates the time when echo signal arrives in the PD1.

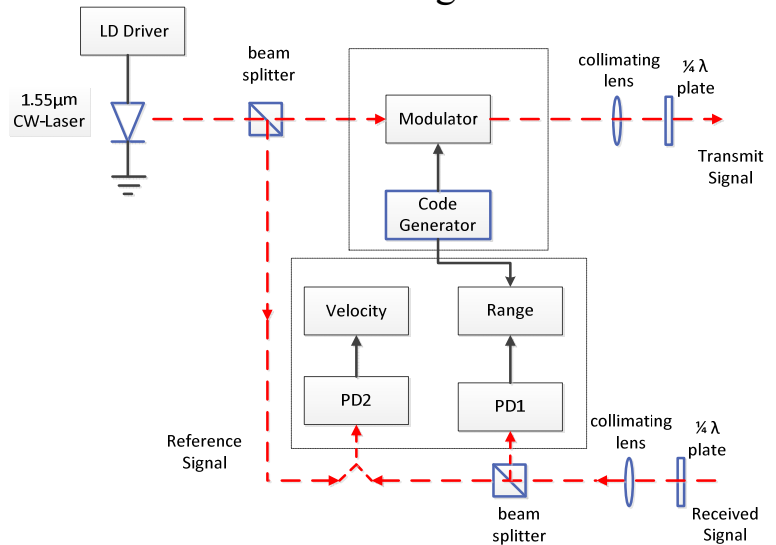

Fig 3 Simulation schematic
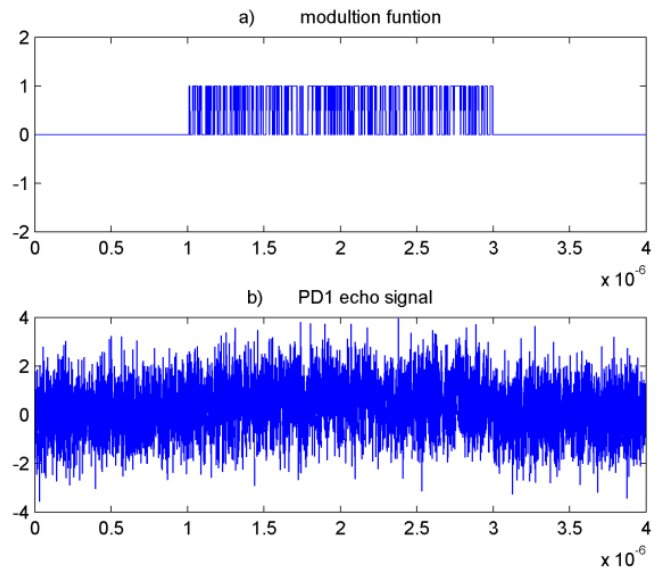

Fig 4 a)modulation function; b)echo signal from PD1 


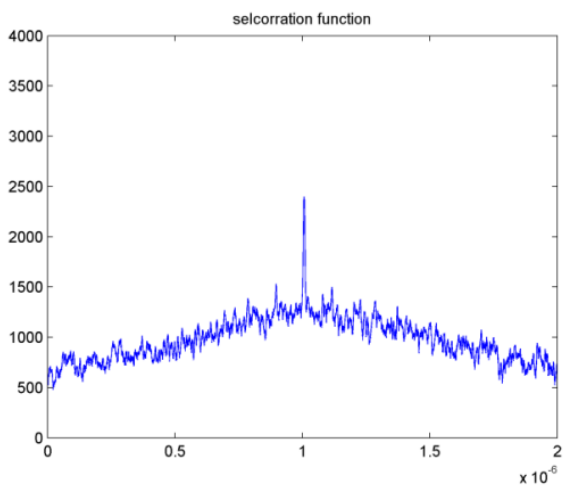

Fig 5 the cross-correlation function between PD1 echo signal and bipolar modulation codes

Figure 6 shows the Doppler echo signal with PN modulation. While Figure 6(a) shows the simulated echo signal without low pass filter, Figure 6(b) shows the echo signal with low pass filtering, which is used to simulate the processing of optical wave passing through atmosphere.

Figure 7 shows the echo signal with Gaussian noise added and the frequency spectrum of the echo signal. The echo has two spectral line of $\pm 3.6 \times 10^{\circ} \mathrm{Hz}$, thus the calculated velocity

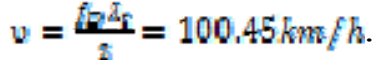
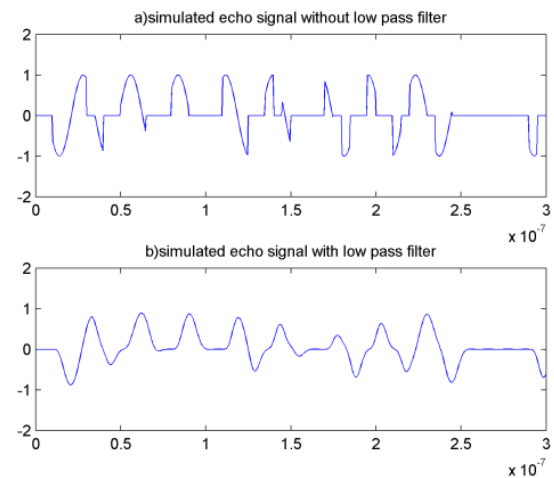

Figure 6(a) the simulated echo signal without low pass filter, (b) the simulated echo signal with low pass filtering
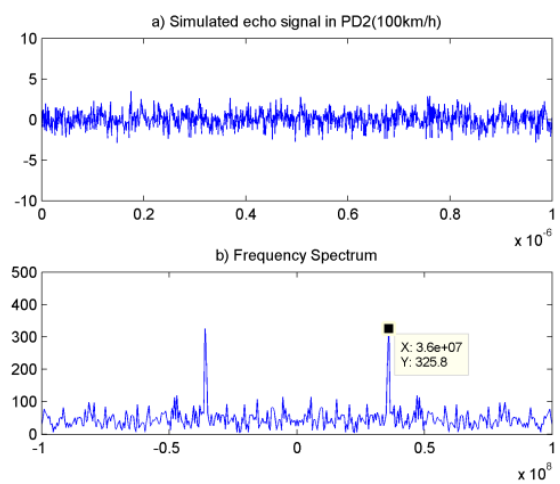

Figure 7(a) the simulated echo signal in PD2 $(100 \mathrm{~km} / \mathrm{s})$, (b) frequency spectrum 


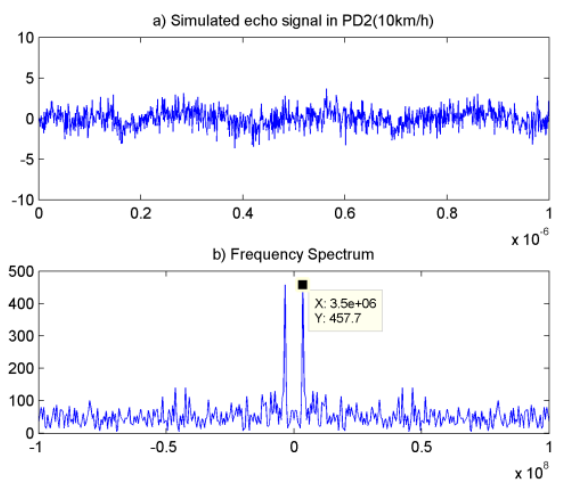

Figure 8(a) the simulated echo signal in PD2 $(10 \mathrm{~km} / \mathrm{s})$, (b) frequency spectrum
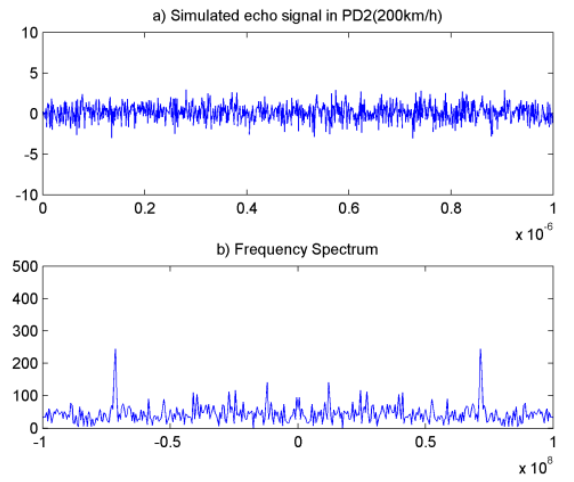

Figure 9(a) the simulated echo signal in PD2 $(200 \mathrm{~km} / \mathrm{s})$, (b) frequency spectrum
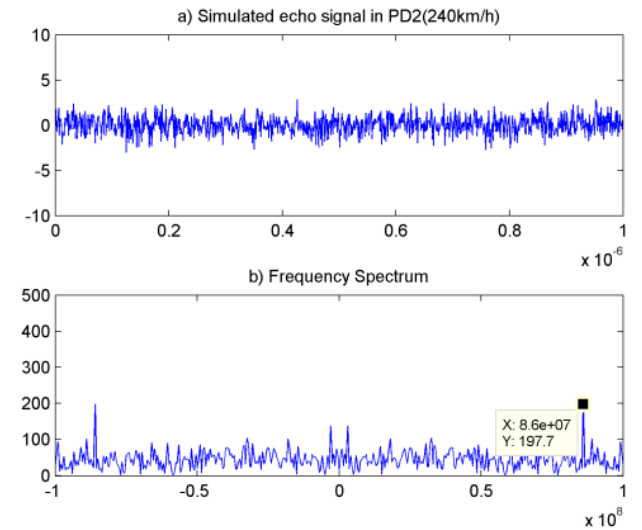

Figure 10(a) the simulated echo signal in PD2 $(240 \mathrm{~km} / \mathrm{s})$, (b) frequency spectrum
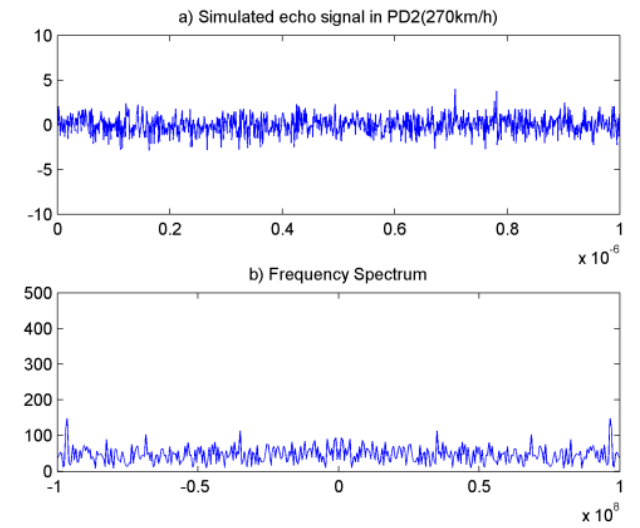

Figure 11(a) the simulated echo signal in PD2 $(270 \mathrm{~km} / \mathrm{s})$, (b) frequency spectrum 
Figure 8- Figure 10 show the conditions when $v=10 \mathrm{~km} / \mathrm{h}, v=200 \mathrm{~km} / \mathrm{h}, v=240 \mathrm{~km} \mathrm{~s}_{\mathrm{h}} \mathrm{h}$ respectively, and the Doppler spectrum lines are obvious. Figure 11 shows the echo Doppler signal when $v=270 \mathrm{~km} / \mathrm{h}$, the spectrum line are not so obvious as the above conditions, for the sampling rate is not high enough for this condition. Fortunately, we do not need to worry about the velocity of more than $240 \mathrm{~km} / \mathrm{h}$ in practice. We have demonstrated the feasibility of range measurement and velocity measurement by the proposed Doppler pulse laser radar. The maximum measurable speed is more than $240 \mathrm{~km} / \mathrm{h}$, and the minimum measurable is less than $10 \mathrm{~km} / \mathrm{h}$, which makes the speed measurement become practical.

\section{Conclusion}

We have demonstrated the viability of range and velocity measurement in Pulse Doppler Laser Radar. We used PN code to modulate the $1.55 \mu \mathrm{m}$ continuous laser in the laser Radar transmitter firstly, and then direction detection was employed to obtain the target range and coherence detection was used to get the target speed simultaneously in the Laser Radar receiver.

Compared to previous method, our method employs PN Code to modulate laser directly in the transmitter of laser Radar, and uses FFT directly to calculate the Doppler frequency, which makes the systems more flexible. The maximum measurable speed can be as high as $240 \mathrm{Km} / \mathrm{h}$ which matches with the sampling theorem. Moreover, the length of the transmitted signal is several microseconds, which makes the possibility of realizing a high scan speed in automotive applications.

\section{Acknowledgments}

This work is supported by Natural Science Foundation of Hubei Province, China, Grant No. 2014 CFB815.

\section{References}

[1] M. J. Halmos, "Eyesafe 3D flash ladar for targets under obscuration," in AeroSense 2003, 2003, pp. 70-83.

[2] J. Beraldin, F. Blais, P. Boulanger, L. Cournoyer, J. Domey, S. El-Hakim, et al., "Real world modelling through high resolution digital 3D imaging of objects and structures," ISPRS Journal of Photogrammetry and Remote Sensing, vol. 55, pp. 230-250, 2000.

[3] X. Mao, D. Inoue, H. Matsubara, and M. Kagami, "Demonstration of In-Car Doppler Laser Radar at 1.55 for Range and Speed Measurement," Intelligent Transportation Systems, IEEE Transactions on, vol. 14, pp. 599-607, 2013.

[4] X. S. Mao, D. Inoue, S. Kato, and M. Kagami, "Amplitude-Modulated Laser Radar for Range and Speed Measurement in Car Applications," Ieee Transactions on Intelligent Transportation Systems, vol. 13, pp. 408-413, Mar 2012.

[5] S. Gao, M. O'Sullivan, and R. Hui, "Complex-optical-field lidar system for range and vector velocity measurement," Opt Express, vol. 20, pp. 25867-75, Nov 52012.

[6] A. Dolfi-Bouteyre, G. Canat, M. Valla, B. Augere, C. Besson, D. Goular, et al., "Pulsed 1.5-m LIDAR for Axial Aircraft Wake Vortex Detection Based on High-Brightness Large-Core Fiber Amplifier," Selected Topics in Quantum Electronics, IEEE Journal of, vol. 15, pp. 441-450, 2009.

[7] M. Harris, R. I. Young, F. Köpp, A. Dolfi, and J.-P. Cariou, "Wake vortex detection and monitoring," Aerospace science and Technology, vol. 6, pp. 325-331, 2002.

[8] J. B. Sherman, "A laser radar ranging system using pseudo-random-code modulation," Education, IEEE Transactions on, vol. 9, pp. 2-9, 1966. 
[9] D. M. Norman and C. S. Gardner, "Satellite laser ranging using pseudonoise code modulated laser diodes," Applied optics, vol. 27, pp. 3650-3655, 1988.

[10] R. D. Richmond and S. C. Cain, Direct-detection LADAR systems: SPIE Press, 2010. 